\title{
Thiazolo[4,5-d]pyrimidines: synthesis and antibacterial evaluation
}

\author{
Mohammad Rahimizadeh',*, Mehdi Bakavoli', \\ Ali Shiri ${ }^{1}$, Reyhaneh Faridnia ${ }^{1}$, Parvaneh Pordeli ${ }^{2}$ \\ and Fatemeh Oroojalian ${ }^{3}$ \\ ${ }^{1}$ Department of Chemistry, School of Sciences, Ferdowsi \\ University of Mashhad, 91775-1436 Mashhad, Iran \\ ${ }^{2}$ Department of Biology, School of Sciences, Ferdowsi \\ University of Mashhad, 91775-1436 Mashhad, Iran \\ ${ }^{3}$ Department of Microbiology, School of Sciences, Isfahan \\ University, Isfahan, Iran \\ *Corresponding author \\ e-mail: rahimizh@yahoo.com
}

2004; Bhattacharya et al., 2005) and vanilloid receptor I TRPVI antagonism (Xi et al., 2005). Also, some thiazolopyrimidines have been found to possess anti-inflammatory and analgesic activities (Russo et al., 1993; Pawan and Sawhney, 1997; Ashok et al., 2007).

Although thiazole ring construction has been reported numerous times and adequately reviewed in the literature (Metwally et al., 2004), there are few reports on the synthesis of fused thiazolopyrimidines and especially thiazolo [4,5-d] pyrimidines. Walek and coworkers (Walek, 1983; Walek et al., 1984) prepared compound (I) by heating the thiazole (II) at $180^{\circ} \mathrm{C}$ for $2 \mathrm{~h}$, whereas Wobig (1989) prepared thiazolopyrimidine derivatives (III) by cyclization of aminothiazoles (IV) with formamide, formic acid, and dimethyl malonate.

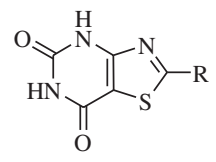

(I)<smiles>[R]c1nc2nc(SC)sc2c(=O)n1[R]</smiles>

(III)<smiles>[R]c1nc(N)c(C(=O)NC(=O)OCC)s1</smiles>

(II)<smiles>[R1]NC(=O)c1sc(C)nc1N</smiles>

(IV)
Keywords: antibacterial evaluation; heterocyclization; thiazolo[4,5- $d]$ pyrimidine.

\section{Introduction}

In medicinal chemistry, pyrimidine derivatives have been known for their therapeutic applications. The presence of a pyrimidine system in thymine, cytosine, and uracil, which are the essential building blocks of nucleic acids, is one possible reason for the activity (Amir et al., 2007). Similarly, thiazole derivatives possess diverse pharmacological activities. Fused derivatives of pyrimidine and thiazole are also bioactive. For example, thiazolopyrimidine derivatives have been the focus of a great deal of interest owing to their antimicrobial (Vicini et al., 2003; Zitouni et al., 2004), antiviral (Sircar et al., 1986; Nagahara et al., 1990; Kini et al., 1991), and antitumor (El-Subbagh and Alobaid, 1996) activities. They have been used as potent and selective inhibitors of acetyl-CoA carboxylase 2 (Clark et al., 2007) and VEGF receptors I and II (Kiselyov et al., 2006). They have also shown potent and selective human adenosine $\mathrm{A}_{3}$ receptor (Van Tilburg et al., 2001; Jung et al.,
Efficient synthesis of various derivatives of thiazolopyrimidine have been reported by the reaction of 4,6-dichloro-5-aminopyrimidine with isothiocyanates (Liu et al., 2005; Lebsack et al., 2009). The reaction of 4-chloro-2,3-dihydrothiazolidine-5-carboxaldehyde with urea in ethanol solution at reflux in the presence of a catalytic amount of $\mathrm{Et}_{3} \mathrm{~N}$ afforded new polysubstituted thiazolopyrimidine derivatives (El Rady, 2008). Akbari et al. (2008) have synthesized 7-aryl-5-thioxo4,5,6,7-tetrahydro-3H-thiazolo[4,5- $d$ ]pyrimidin-2-ones by the reaction of 2,4-thiazolidine with thiourea and different aromatic aldehydes.

Taking into account these findings and in continuation of our efforts to develop novel and efficient routes to heterocyclic derivatives of pyrimidine with potential biological turned our attention to the synthesis of various new derivatives of thiazolo[4,5- $d$ ]pyrimidine. It was of interest to investigate antibacterial activity of the new compounds against Staphylococcus aureus PTCC 1431, Bacillus subtilis PTCC 1365, Escherichia coli HB101 BA 7601C, and Pseudomonas aeruginosa PTCC 1074 bacteria. activities (Bakavoli et al., 2008, 2009, 2010a, b, 2011a, b), we 


\section{Results and discussion}

\section{Chemistry}

5-Bromo-2,4-dichloro-6-methylpyrimidine, which was prepared as reported by us previously (Bakavoli et al., 2006), was treated with ammonia in ethanol at room temperature to give 4-amino-5-bromo-2-chloro-6-methyl-pyrimidine (1). The heterocyclization of compound $\mathbf{1}$ with carbon disulfide in the presence of $\mathrm{KOH}$ at room temperature quantitatively afforded 5-chloro-7-methyl-2,3-dihydropyrimido[4,5- $d][1,3]$ thiazol-2thione (2). The ${ }^{1} \mathrm{H}$ NMR spectrum of this compound shows a singlet at $\delta 2.52 \mathrm{ppm}$ and a broad singlet at $\delta 3.85$ for the methyl and $\mathrm{NH}$ groups of the compound $\mathbf{2}$, respectively. In the mass spectra of this compound, molecular ion peak is observed at $m / z$ 217, which corresponds to the desired molecular formula. To prepare new derivatives of thiazolo [4,5- $d$ ] pyrimidine, compound $\mathbf{2}$ was treated with various alkyl halides bearing different functional groups in the presence of $\mathrm{Et}_{3} \mathrm{~N}$ in boiling acetonitrile. The desired new derivatives of thiazolo[4,5- $d]$ pyrimidine $\mathbf{3 a - f}$ were obtained in good to excellent yields (Scheme 1).

The structures of the synthesized compounds $\mathbf{3}$ were determined by the spectral and microanalytical data. For example, the ${ }^{1} \mathrm{H}$ NMR spectrum of compound $\mathbf{3 a}$ does not exhibit a signal for an $\mathrm{NH}$ moiety that is present at $\delta 3.85$ in the spectrum of the precursor 2. Product 3a shows two sharp singlets at $\delta 2.55$ and $\delta 2.88$ for two methyl groups. The IR spectrum of $\mathbf{3 a}$ does not show the stretching vibration band at $3280 \mathrm{~cm}^{-1}$ for the $\mathrm{NH}$ group that is found in the IR spectrum to the $\mathrm{NH}$ group of the precursor 2. Instead, a new $\mathrm{C}=\mathrm{N}$ stretching vibration band at $1640 \mathrm{~cm}^{-1}$ is observed. The molecular ion peaks in the mass spectrum of compound $\mathbf{3 a}$ are observed at $\mathrm{m} / \mathrm{z}, 231$ and 233, which is consistent with the presence of one chlorine atom in the molecule. These results, together with the results of microanalysis fully support the molecular formula of $\mathrm{C}_{7} \mathrm{H}_{6} \mathrm{ClN}_{3} \mathrm{~S}_{2}$ for $\mathbf{3 a}$.
A nucleophilic substitution of the chlorine atom at the 5-position of the pyrimidine ring of the synthesized compounds 3a-f with morpholine yielded the corresponding morpholinesubstituted derivatives $\mathbf{4 a - f}$ in good yields. The ${ }^{1} \mathrm{H}$ NMR spectra of all new compounds $\mathbf{4 a - f}$ exhibit the characteristic signals of the morpholine unit, which along with other data unambiguously confirm the desired structures of compounds $\mathbf{4 a - f}$.

\section{Antibacterial evaluation}

The tested microorganisms were Gram-positive and Gramnegative bacteria. The sensitivity of the selected microorganisms, S. aureus PTCC 1431, B. subtilis PTCC 1365, E. coli HB101 BA 7601C, and P. aeruginosa PTCC 1074, to all synthesized compounds 3a-f and $\mathbf{4 a - f}$ was determined in vitro. The compounds were dissolved in DMSO and the tests were carried out using a disk diffusion method (Reeves and White, 1983). Streptomycin was used as a reference bactericidal antibiotic (Table 1). The evaluations were obtained in triplicate and the results with differences greater than $5 \%$ were discarded, and the measurements repeated. It can be concluded from the data in Table 1 that all compounds $\mathbf{3 a - f}$ and $\mathbf{4 a - f}$ are highly active against $B$. subtilis and $P$. aeruginosa. A slightly lower activity is observed against $S$. aureus. Compounds $\mathbf{3 c}, \mathbf{3 d}$, and $\mathbf{4 b}$ are highly active against E. coli, which is a Gram-negative bacteria.

\section{Conclusion}

In summary, a new series of thiazolo[4,5- $d$ ]pyrimidine derivatives were synthesized and their antibacterial activities evaluated. Many compounds proved to be more active against $B$. subtilis and $P$. aeruginosa than streptomycin, the reference

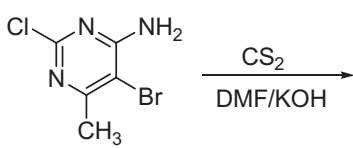

(1)<smiles>Cc1nc(Cl)nc2[nH]c(=S)sc12</smiles>

(2)<smiles>[R]C[N+](C)(C)C</smiles>

(3a-f)

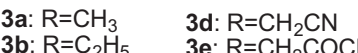

3b: $\mathrm{R}=\mathrm{C}_{2} \mathrm{H}_{5} \quad$ 3e: $\mathrm{R}=\mathrm{CH}_{2} \mathrm{COCH}_{3}$

3c: $\mathrm{R}=\mathrm{CH}_{2} \mathrm{Ph} \quad$ 3f: $\mathrm{R}=\mathrm{CH}_{2} \mathrm{CO}_{2} \mathrm{Et}$

Morpholine

$\mathrm{Et}_{3} \mathrm{~N}, \mathrm{EtOH}$

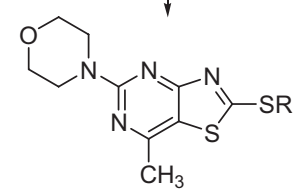

(4a-f)

4a: $\mathrm{R}=\mathrm{CH}_{3} \quad$ 4d: $\mathrm{R}=\mathrm{CH}_{2} \mathrm{CN}$

4b: $\mathrm{R}=\mathrm{C}_{2} \mathrm{H}_{5} \quad$ 4e: $\mathrm{R}=\mathrm{CH}_{2} \mathrm{COCH}_{3}$

4c: $\mathrm{R}=\mathrm{CH}_{2} \mathrm{Ph}$ 4f: $\mathrm{R}=\mathrm{CH}_{2} \mathrm{CO}_{2} \mathrm{Et}$

Scheme 1 Synthesis of thiazolo[4,5- $d]$ pyrimidines. 
Table 1 Antibacterial data for compounds $\mathbf{3 a - f}$ and $\mathbf{4 a - f}$.

\begin{tabular}{|c|c|c|c|c|}
\hline \multirow[t]{2}{*}{ Compound } & \multicolumn{2}{|c|}{ Gram-negative bacteria } & \multicolumn{2}{|l|}{ Gram-positive bacteria } \\
\hline & $\begin{array}{l}\text { Escherichia coli } \\
\text { HB101 BA 7601C }\end{array}$ & $\begin{array}{l}\text { Pseudomonas aeruginosa } \\
\text { PTCC } 1074\end{array}$ & $\begin{array}{l}\text { Staphylococcus aureus } \\
\text { PTCC } 1431\end{array}$ & $\begin{array}{l}\text { Bacillus subtilis } \\
\text { PTCC } 1365\end{array}$ \\
\hline $3 \mathbf{a}$ & $15^{\mathrm{a}}(-)^{\mathrm{b}, \mathrm{c}}$ & $14(++)$ & $12(-)$ & $15(++)$ \\
\hline $3 \mathbf{b}$ & $17(+)$ & $15(++)$ & $12(-)$ & $15(++)$ \\
\hline $3 \mathbf{c}$ & $18(++)$ & $15.5(++)$ & $14(-)$ & $14(++)$ \\
\hline 3d & $18(++)$ & $13(++)$ & $14(-)$ & $14(++)$ \\
\hline $3 \mathbf{e}$ & $17(+)$ & $14(++)$ & $14(-)$ & $13(++)$ \\
\hline 3f & $17(+)$ & $15(++)$ & $13(-)$ & $14(++)$ \\
\hline $4 a$ & $15(-)$ & $15.3(++)$ & $12(-)$ & $14(++)$ \\
\hline $4 b$ & $20(++)$ & $15(++)$ & $13(-)$ & $14.5(++)$ \\
\hline $4 c$ & $13(-)$ & $15.5(++)$ & $12(-)$ & $15(++)$ \\
\hline $4 d$ & $14(-)$ & $16(++)$ & $15(+)$ & $14(++)$ \\
\hline $4 e$ & $15(-)$ & $13(++)$ & $13(-)$ & $13.5(++)$ \\
\hline 4f & $17(+)$ & $9(-)$ & $14.5(-)$ & $14.3(++)$ \\
\hline Streptomycin (standard) & 17 & 10 & 15 & 10 \\
\hline
\end{tabular}

a Zones of inhibition in millimeters.

b (++) highly sensitive; (+) moderately sensitive; (-) slightly sensitive.

${ }^{\mathrm{c}}$ The maximum inhibition zone for each compound has been shown. Discs of each concentration were placed in triplicate in Muller-Hinton agar medium seeded with fresh bacteria separately and the average was reported.

antibiotic. Hence, it is concluded that there is a promising scope for further development in this field.

\section{Experimental}

Melting points were recorded on an electrothermal type 9100 melting point apparatus and are not corrected. The IR spectra were obtained in $\mathrm{KBr}$ pellets on a Thermo Nicolet Avatar 370-FTIR spectrometer. The ${ }^{1} \mathrm{H}$ NMR (100 MHz) spectra were recorded on a Bruker AC 100 spectrometer in $\mathrm{CDCl}_{3}$ solution with TMS as an internal reference. The electron impact mass spectra were obtained on a Varian Mat $\mathrm{CH}-7$ instrument at $70 \mathrm{eV}$. Elemental analysis was performed on a Thermo Finnigan Flash EA 1112 instrument.

\section{5-Chloro-7-methylthiazolo[4,5- $d]$ pyrimidine- 2(3H)-thione (2)}

To a magnetically stirred solution of 4-amino-5-bromo-2-chloro-6methyl-pyrimidine (1) (1 mmol, $0.22 \mathrm{~g}$ ) and carbon disulfide $(0.1 \mathrm{ml})$ in dry DMF $(5 \mathrm{ml})$, powdered $\mathrm{KOH}(1 \mathrm{mmol}, 0.056 \mathrm{~g})$ was added, and the mixture stirred for $2 \mathrm{~h}$ at room temperature. Then, the mixture was heated at $70-80^{\circ} \mathrm{C}$ for $0.5 \mathrm{~h}$. After cooling, water $(10 \mathrm{ml})$ was added and the solution was neutralized with acetic acid. The resulting precipitate was filtered off and crystallized from ethanol: yield 80\%; mp $197^{\circ} \mathrm{C}$ (decomp.); ${ }^{1} \mathrm{H}$ NMR: $\delta 2.52$ (s, 3H, $\mathrm{CH}_{3}$-pyrimidine), 3.85 (s, 1H, NH, D O exchangeable); IR: $v$ 3010, $1233(\mathrm{C}=\mathrm{S}) \mathrm{cm}^{-1}$; MS: $\mathrm{m} / \mathrm{z}, 217\left(\mathrm{M}^{+}\right.$for $\left.{ }^{35} \mathrm{Cl}\right), 219\left(\mathrm{M}^{+}\right.$for $\left.{ }^{37} \mathrm{Cl}\right)$. Analysis calculated for $\mathrm{C}_{6} \mathrm{H}_{5} \mathrm{ClN}_{3} \mathrm{~S}_{2}$ : C, 33.00; $\mathrm{H}, 1.85 ; \mathrm{N}, 19.30 ; \mathrm{S}, 29.45$. Found: C, 29.91; $\mathrm{H}, 2.71 ; \mathrm{N}, 16.95 ; \mathrm{S}, 21.54$.

\section{General procedure for the synthesis of thiazolo[4,5- $d]$ pyrimidines $3 a-f$}

To a solution of 5-chloro-7-methyl-2,3-dihydropyrimido[4,5- $d][1,3]$ thiazol-2-thione (1) $(1 \mathrm{mmol}, 0.21 \mathrm{~g})$ and $\mathrm{Et}_{3} \mathrm{~N}(1 \mathrm{mmol}, 0.1 \mathrm{~g})$ in $\mathrm{CH}_{3} \mathrm{CN}(15 \mathrm{ml})$, the appropriate alkyl halide $(1.1 \mathrm{mmol})$ was added, and the solution was heated under reflux for $2-3 \mathrm{~h}$. The progress of the reaction was monitored by TLC using $n$-hexane/EtOAc (6:4). Then, the solvent was removed on a rotary evaporator and the resulting solid was crystallized from methanol.

5-Chloro-7-methyl-2-(methylthio)thiazolo[4,5- $d]$ pyrimidine (3a) Yield 60\%; mp 137-144 ${ }^{\circ} \mathrm{C}$; ${ }^{1} \mathrm{H}$ NMR: $\delta 2.55$ (s, 3H, $\mathrm{CH}_{3}-$ pyrimidine), 2.88 (s, $3 \mathrm{H},-\mathrm{SCH}_{3}$ ); IR: $v 3010,1650 \mathrm{~cm}^{-1}$; MS: $\mathrm{m} / z, 231$ $\left(\mathrm{M}^{+}\right), 233\left(\mathrm{M}^{+}+2\right)$. Analysis calculated for $\mathrm{C}_{7} \mathrm{H}_{6} \mathrm{ClN}_{3} \mathrm{~S}$ : $\mathrm{C}, 36.28 ; \mathrm{H}$, $2.61 ; \mathrm{N}, 18.13$; S, 27.67. Found: C, 36.01; H, 2.57; N, 18.02; S, 27.44.

5-Chloro-2-(ethylthio)-7-methylthiazolo[4,5- $d$ ]pyrimidine (3b) Yield 65\%; mp $107^{\circ} \mathrm{C}$; ${ }^{1} \mathrm{H}$ NMR: $\delta 1.53$ (t, 3H, $\mathrm{CH}_{3}$ ), 2.70 (s, $3 \mathrm{H}, \mathrm{CH}_{3}$-pyrimidine), 3.43 (q, $2 \mathrm{H},-\mathrm{SCH}_{2}$ ); IR: $v 3015,1630 \mathrm{~cm}^{-1}$; MS: $m / z 245\left(\mathrm{M}^{+}\right), 247\left(\mathrm{M}^{+}+2\right)$. Analysis calculated for $\mathrm{C}_{8} \mathrm{H}_{8} \mathrm{ClN}_{3} \mathrm{~S}_{2}$ : C, 39.10; H, 3.28; N, 17.10; S, 26.10. Found: C, 38.88; H, 3.20; N, $16.90 ; \mathrm{S}, 25.78$.

2-(Benzylthio)-5-chloro-7-methylthiazolo[4,5- $d]$ pyrimidine (3c) Yield 70\%; mp 98-100 ${ }^{\circ}$; ${ }^{1} \mathrm{H}$ NMR: $\delta 2.70\left(\mathrm{~s}, 3 \mathrm{H}, \mathrm{CH}_{3}\right), 4.71$ (s, 2H, $\mathrm{CH}_{2}$ ), 7.31-7.62 (m, 5H, phenyl); IR: v 3000, $1660 \mathrm{~cm}^{-1}$; MS: $\mathrm{m} / 2,307\left(\mathbf{M}^{+}\right), 309\left(\mathbf{M}^{+}+2\right)$. Analysis calculated for $\mathrm{C}_{13} \mathrm{H}_{10} \mathrm{ClN}_{3} \mathrm{~S}_{2}$ : C, 50.72; H, 3.27; N, 13.65; S, 20.83. Found: C, 50.66; H, 3.16; N, $13.57 ;$ S, 20.69 .

2-(5-Chloro-7-methylthiazolo[4,5-d]pyrimidin-2-ylthio) acetonitrile (3d) Yield 63\%; mp $104-110^{\circ} \mathrm{C} ;{ }^{1} \mathrm{H}$ NMR: $\delta 2.78(\mathrm{~s}, 3 \mathrm{H}$, $\mathrm{CH}_{3}$ ), 4.32 (s, 2H, $\mathrm{CH}_{2}$ ); IR: v 2990, 2215, $1640 \mathrm{~cm}^{-1} ; \mathrm{MS}: \mathrm{m} / \mathrm{z}, 256$ $\left(\mathrm{M}^{+}\right), 258\left(\mathrm{M}^{+}+2\right)$. Analysis calculated for $\mathrm{C}_{8} \mathrm{H}_{5} \mathrm{ClN}_{4} \mathrm{~S}_{2}: \mathrm{C}, 37.43 ; \mathrm{H}$, $1.96 ; \mathrm{N}, 21.82 ; \mathrm{S}, 24.98$. Found: C, 37.22; H, 1.88; N, 21.90; S, 24.89.

1-(5-Chloro-7-methylthiazolo[4,5- $d$ ]pyrimidin-2-ylthio) propan2-one (3e) Yield 73\%; mp $114^{\circ} \mathrm{C}$; ${ }^{1} \mathrm{H}$ NMR: $\delta 2.41\left(\mathrm{~s}, 3 \mathrm{H}, \mathrm{CH}_{3}\right.$ ), 2.82 (s, 3H, $\mathrm{CH}_{3}$-pyrimidine), 4.41 (s, $2 \mathrm{H},-\mathrm{SCH}_{2}$ ); IR: v 3010, 1710 , $1660 \mathrm{~cm}^{-1}$; MS: $\mathrm{m} / z, 273\left(\mathrm{M}^{+}\right), 275\left(\mathrm{M}^{+}+2\right)$. Analysis calculated for $\mathrm{C}_{9} \mathrm{H}_{8} \mathrm{ClN}_{3} \mathrm{OS}_{2}$ : C, 39.49; H, 2.95; N, 15.35; S, 23.43. Found: C, $39.51 ; \mathrm{H}, 2.92 ; \mathrm{N}, 15.18 ; \mathrm{S}, 23.35$. 
Ethyl-2-(5-chloro-7-methylthiazolo[4,5-d]pyrimidin-2-ylthio) acetate (3f) Yield 58\%; mp $109^{\circ} \mathrm{C} ;{ }^{1} \mathrm{H}$ NMR: $\delta 1.38\left(\mathrm{t}, 3 \mathrm{H}, \mathrm{CH}_{3}\right)$, 2.73 (s, 3H, $\mathrm{CH}_{3}$-pyrimidine), 4.31 (q, 2H, $-\mathrm{SCH}_{2}$ ); IR: v 3010, 1680, $1630 \mathrm{~cm}^{-1}$; MS: $\mathrm{m} / z 303\left(\mathrm{M}^{+}\right), 305\left(\mathrm{M}^{+}+2\right)$. Analysis calculated for $\mathrm{C}_{10} \mathrm{H}_{10} \mathrm{ClN}_{3} \mathrm{O}_{2} \mathrm{~S}_{2}$ : C, 39.54; H, 3.32; N, 13.83; S, 21.11. Found: C, 39.45; H, 3.29; N, 13.78; S, 20.79.

\section{General procedure for the substitution of chlorine atom in 5-position with morpholine (4a-f)}

To a solution of compounds (3a-f) $(1 \mathrm{mmol})$ and morpholine $(1.1 \mathrm{mmol}, 0.088 \mathrm{~g})$ in ethanol $(10 \mathrm{ml}), \mathrm{Et}_{3} \mathrm{~N}(1.1 \mathrm{mmol}, 0.1 \mathrm{~g})$ was added and the solution was heated for approximately $8 \mathrm{~h}$. The solvent of the reaction was reduced to half of the initial volume. The resulting solid was filtered off and recrystallized in ethanol.

7-Methyl-2-(methylthio)-5-morpholinothiazolo[4,5-d]pyrimidine (4a) Yield 64\%; mp 204-205 ${ }^{\circ} \mathrm{C} ;{ }^{1} \mathrm{H}$ NMR: $\delta 2.52$ (s, 3H, $\mathrm{CH}_{3}$ ), 2.83 (s, 3H, $\mathrm{CH}_{3}$-pyrimidine), 3.71-3.92 (m, 8H, morpholine); IR: v 3020, $1610 \mathrm{~cm}^{-1}$; MS: $\mathrm{m} / z 282\left(\mathrm{M}^{+}\right)$. Analysis calculated for $\mathrm{C}_{11} \mathrm{H}_{14} \mathrm{~N}_{4} \mathrm{OS}_{2}$ : C, 46.79; H, 5.00; N, 19.84; S, 22.71. Found: C, $46.70 ; \mathrm{H}, 4.97 ; \mathrm{N}, 19.78 ; \mathrm{S}, 22.64$

2-(Ethylthio)-7-methyl-5-morpholinothiazolo[4,5-d]pyrimidine (4b) Yield 50\%; mp 129-131 ${ }^{\circ} \mathrm{C}$; ${ }^{1} \mathrm{H}$ NMR: $\delta 1.47$ (t, 3H, $\mathrm{CH}_{3}$ ), 2.51 (s, 3H, $\mathrm{CH}_{3}$ ), 3.43 (q, 2H, - $\mathrm{SCH}_{2}$ ), 3.71-4.05 (m, 8H, morpholine); IR: $v$ 3030, $1650 \mathrm{~cm}^{-1}$; MS: $\mathrm{m} / z, 296\left(\mathrm{M}^{+}\right)$. Analysis calculated for $\mathrm{C}_{12} \mathrm{H}_{16} \mathrm{~N}_{4} \mathrm{OS}_{2}$ : C, 48.62; H, 5.44; N, 18.90; S, 21.64. Found: C, $48.52 ; \mathrm{H}, 5.41 ; \mathrm{N}, 18.86 ; \mathrm{S}, 21.53$.

2-(Benzylthio)-7-methyl-5-morpholinothiazolo[4,5- $d$ ]pyrimidine (4c) Yield 55\%; mp 143-145 ${ }^{\circ} \mathrm{C} ;{ }^{1} \mathrm{H}$ NMR: $\delta 2.51(\mathrm{~s}, 3 \mathrm{H}$, $\mathrm{CH}_{3}$ ), 3.72-4.03 (m, 8H, morpholine), 4.71 (s, 2H, $-\mathrm{SCH}_{2}$ ), 7.317.52 (m, 5H, phenyl); IR: v 3050, $1630 \mathrm{~cm}^{-1}$; MS: $\mathrm{m} / \mathrm{z}, 358(\mathrm{M}+)$. Analysis calculated for $\mathrm{C}_{17} \mathrm{H}_{18} \mathrm{~N}_{4} \mathrm{OS}_{2}$ : C, 56.96; H, 5.06; N, 15.63; S, 17.89. Found: C, 56.89; H, 5.01; N, 15.56; S, 17.80 .

2-(7-Methyl-5-morpholinothiazolo[4,5-d]pyrimidin-2-ylthio) acetonitrile (4d) Yield 48\%; mp 185-189 ${ }^{\circ} \mathrm{C} ;{ }^{1} \mathrm{H}$ NMR: $\delta 2.48(\mathrm{~s}$, $3 \mathrm{H}, \mathrm{CH}_{3}$ ), 3.72-3.91 (m, 8H, morpholine), 4.33 (s, 2H, - $\mathrm{SCH}_{2}$ ); IR: $v$ 3030, 2215, $1650 \mathrm{~cm}^{-1}$; MS: $\mathrm{m} / z, 307\left(\mathrm{M}^{+}\right)$. Analysis calculated for $\mathrm{C}_{12} \mathrm{H}_{13} \mathrm{~N}_{5} \mathrm{OS}_{2}$ : C, 46.89; $\mathrm{H}, 4.26 ; \mathrm{N}, 22.78 ; \mathrm{S}, 20.86$. Found: $\mathrm{C}$, $46.75 ; \mathrm{H}, 4.22 ; \mathrm{N}, 22.77 ; \mathrm{S}, 20.79$.

1-(7-Methyl-5-morpholinothiazolo[4,5-d]pyrimidin-2-ylthio) propan-2-one (4e) Yield $43 \%$; $\mathrm{mp} 185-189^{\circ} \mathrm{C}$; ${ }^{1} \mathrm{H}$ NMR: $\delta 2.48$ (s, $3 \mathrm{H}, \mathrm{CH}_{3}$ ), 2.72 (s, $3 \mathrm{H}, \mathrm{CH}_{3}$ ), 3.72-3.93 (m, 8H, morpholine), 4.46 (s, 2H, -SCH $\mathrm{SCH}_{2}$; IR: v 3010, 1720, $1640 \mathrm{~cm}^{-1}$; MS: $\mathrm{m} / \mathrm{z} 324\left(\mathrm{M}^{+}\right)$. Analysis calculated for $\mathrm{C}_{13} \mathrm{H}_{16} \mathrm{~N}_{4} \mathrm{O}_{2} \mathrm{~S}_{2}$ : C, 48.13; H, 4.97; N, 17.27; S, 19.77. Found: C, 48.12; H, 4.95; N, 17.25; S, 19.75 .

Ethyl2-(7-methyl-5-morpholinothiazolo[4,5-d]pyrimidin-2ylthio)acetate (4f) Yield $43 \%$; mp $173-180{ }^{\circ} \mathrm{C}$; ${ }^{1} \mathrm{H}$ NMR: $\delta 1.32$ (s, $\left.3 \mathrm{H}, \mathrm{CH}_{3}\right), 2.61$ (s, $\left.2 \mathrm{H},-\mathrm{SCH}_{2}\right), 3.82(\mathrm{~m}, 8 \mathrm{H}$, morpholine), 4.27 (s, 2H, - $\mathrm{OCH}_{2}$ ). IR: v 3030, 1690, $1630 \mathrm{~cm}^{-1}$. MS: $\mathrm{m} / z, 354\left(\mathrm{M}^{+}\right)$. Analysis calculated for $\mathrm{C}_{14} \mathrm{H}_{18} \mathrm{~N}_{4} \mathrm{O}_{3} \mathrm{~S}_{2}$ : C, 47.44; H, 5.12; N, 15.81; S, 18.09. Found: C, 47.39; H, 5.04; N, 15.69; S, 17.89.

\section{Acknowledgments}

Financial support for this work by the Research Affairs of Ferdowsi University of Mashhad is gratefully acknowledged.

\section{References}

Akbari, J. D.; Tala, S. D.; Dhaduk, M. F.; Joshi, H. S.; Mehta, K. B.; Pathak, S. J. Synthesis of some new pyrazolo $[3,4-d]$ pyrimidines and thiazolo[4,5- $d]$ pyrimidines and evaluation of their antimicrobial activities. Phosphorus Sulfur Silicon 2008, 183, 1471-1477.

Amir, A.; Javed, S. A.; Kumar, H. Pyrimidines as anti-inflammatory agents: a review. Indian J. Pharm. Sci. 2007, 69, 337-343.

Ashok, K.; Chatrasal, S. R.; Sudhir, K. B. Synthesis of 3-[4'(p-chlorophenyl)-thiazol-2'-yl]-2-[(substituted azetidinone/ thiazolidinone)-aminomethyl]-6-bromoquinazolin-4-ones as anti-inflammatory agent. Bioorgan. Med. Chem. 2007, 15, 3089-3096.

Bakavoli, M.; Nikpour, M.; Rahimizadeh, M. New access to thiazolo[4,5- $d$ pyrimidine derivatives. J. Heterocyclic Chem. 2006, 43, 1327-1329.

Bakavoli, M.; Rahimizadeh, M.; Shiri, A.; Eshghi, H.; Nickpour, M. Facile synthesis of 2-anilino-pyrimido[4,5-e][1,3,4] thiadiazines. Heterocycles 2008, 75, 1745-1748.

Bakavoli, M.; Bagherzadeh, G.; Vaseghifar, M.; Shiri, A.; Pordeli, P. Iodine catalyzed synthesis and antibacterial evaluation of new derivatives of thieno[2,3-d]pyrimidine. J. Chem. Res. 2009, 9, 653-655.

Bakavoli, M.; Bagherzadeh, G.; Vaseghifar, M.; Shiri, A.; Pordel, M.; Mashreghi, M.; Pordeli, P.; Araghi, M. Molecular iodine promoted synthesis of new pyrazolo[3,4-d]pyrimidine derivatives as potential antibacterial agents. Eur. J. Med. Chem. 2010a, 45, 647-650.

Bakavoli, M.; Rahimizadeh, M.; Shiri, A.; Akbarzadeh, M.; Mousavi, S. H.; Atapour-Mashhad, H.; Tayarani-Najaran, Z. Synthesis and anticancer evaluation of new derivatives of 3-phenyl-1,5-dimethyl-1H-[1,2,4]triazolo[4', $\left.3^{\prime}: 1,2\right]$ pyrimido[4,5-e][1,3,4]oxadiazine. J. Chem. Res. 2010b, 34, 403-406.

Bakavoli, M.; Rahimizadeh, M.; Shiri, A.; Akbarzadeh, M.; Mousavi, S. H.; Tayarani-Najaran, Z.; Atapour-Mashhad, H.; Nikpour, M. Synthesis of new derivatives of 3-aryl-1,5-dimethyl- $1 H$-[1,2,4] triazolo[ $\left[4^{\prime}, 3^{\prime}: 1,2\right]$ pyrimido[ $\left.4,5-e\right][1,3,4]$ oxadiazines as potential antiproliferative agents. J. Heterocyclic Chem. 2011a, 48, 183187.

Bakavoli, M.; Rahimizadeh, M.; Shiri, A.; Eshghi, H.; Pordeli, P.; Pordel, M.; Nikpour, M. Synthesis and antibacterial evaluations of new pyridazino[4,3-e][1,3,4]oxadiazines. J. Heterocyclic Chem. 2011b, 48, 149-152.

Bhattacharya, P.; Leonard, T. J.; Roy, K. Exploring QSAR of thiazole and thiadiazole derivatives as potent and selective human adenosine A3 receptor antagonists using FA and GFA techniques. Bioorgan. Med. Chem. 2005, 13, 1159-1165.

Clark, F. R.; Zhang, T.; Wang, X.; Wang, R.; Zhang, X.; Camp, S. H.; Beutel, B. A.; Sham, H. L.; Gu, G. Y. Phenoxy thiazole derivatives as potent and selective acetyl-CoA carboxylase 2 inhibitors: modulation of isozyme selectivity by incorporation of phenyl ring substituents. Bioorg. Med. Chem. Lett. 2007, 17, 1961-1965.

El Rady, E. A. A convenient and facile synthesis of new thiazole derivatives. Phosphorus Sulfur Silicon 2008, 183, 1659-1671.

El-Subbagh, I.; Alobaid, A. M. 2,4-Disubstituted thiazoles II. A novel class of antitumor agents, synthesis and biological evaluations. Eur. J. Med. Chem. 1996, 31, 1017-1021.

Jung, Y. K.; Kim, K. S.; Geo, G. Z.; Gross, A. S.; Melman, N.; Jacobson, K. A.; Kim, C. Y. Structure-activity relationships of thiazole and thiadiazole derivatives as potent and selective human adenosine A3 receptor antagonists. Bioorgan. Med. Chem. 2004, 12, 613-623. 
Kini, G. D.; Anderson, J. D.; Sanghvi, Y. S.; Lewis, A. F.; Smee, D. F.; Revankar, G. R.; Robins, R. K.; Cottam, H. B. Synthesis and antiviral activity of certain guanosine analogs in the thiazolo[4,5-d]pyrimidine ring system. J. Med. Chem. 1991, 34, 3006-3010.

Kiselyov, A. S.; Piatanitski, E.; Semenova, M.; Semenov, V. V. $\mathrm{N}$-(Aryl)-4-(azolylethyl)thiazole-5-carboxamides: novel potent inhibitors of VEGF receptors I and II. Bioorg. Med. Chem. Lett. 2006, 16, 602-606.

Lebsack, A. D.; Branstetter, B. J.; Hack, M. D.; Xiao, W.; Peterson, M. L.; Nasser, N.; Maher, M. P.; Ao, H.; Bhattacharya, A.; Kansagara, M.; et al. Identification and synthesis of 2,7-diaminothiazolo[5,4- $d]$ pyrimidine derivatives as TRPV1 antagonists. Bioorg. Med. Chem. Lett. 2009, 19, 40-46.

Liu, J.; Patch, R. J.; Schubert, C.; Player, M. R. Single-step syntheses of 2-amino-7-chlorothiazolo[5,4- $d$ ]pyrimidines: intermediates for bivalent thiazolopyrimidines. J. Org. Chem. 2005, 70, 10194-10197.

Metwally, M. A.; Abdel-latif, E.; Amer, F. A.; Kaupp, G. Versatile 2-amino-4-substituted-1,3-thiazoles: synthesis and reactions. $J$. Sulfur Chem. 2004, 25, 63-85.

Nagahara, K.; Anderson, J. D.; Kini, G. D.; Dalley, N. K.; Larson, S. B.; Smee, D. F.; Jin, A.; Sharma, B. S.; Jolley, W. B. Thiazolo[4,5-d]pyrimidine nucleosides. The synthesis of certain 3- $\beta$-D-ribofuranosyl thiazolo[4,5- $d]$ pyrimidines as potential immunotherapeutic agents. J. Med. Chem. 1990, 33, 407-415.

Pawan, K. S.; Sawhney, S. N. Potent anti-inflammatory 3-thiazole-4(5)-acetic acids of 1,2-benzisothiazole. Bioorg. Med. Chem. Lett. 1997, 7, 2427-2430.

Reeves, D. S.; White, L. O. Principles of Methods of Assaying Antibiotic in Pharmaceutical Microbiology; $3^{\text {rd }}$ Edition. Blackwell: Oxford, 1983, pp. 140-162.

Russo, F.; Guccione, S.; Romeo, G.; Barretta, C. U.; Pucci, S.; Caruso, A.; Amico-Roxas, M.; Cutuli, V. Pyrazolothiazolopyrimidine derivatives as a novel class of anti-inflammatory or antinociceptive agents: synthesis, structural characterization and pharmacological evaluation. Eur. J. Med. Chem. 1993, 28, 363-376.

Sircar, J. C.; Suto, M. J.; Scott, M. E.; Dong, M. K.; Gilbertsen, R. B. Inhibitors of human purine nucleoside phosphorylase. Synthesis, purine nucleoside phosphorylase inhibition, and T-cell cytotoxicity of 2,5-diaminothiazolo[5,4- $d]$ pyrimidin-7$(6 H)$-one and 2,5-diaminothiazolo[4,5-d]pyrimidin-7(6H)-one. Two thioisosteres of 8-aminoguanine. J. Med. Chem. 1986, 29, 1804-1806.

Van Tilburg, E. W.; Van der Klein, P.A.; De Groote, M.; Beukers, M.W.; Wzerman, A. P. Substituted 4-phenyl-2-(phenylcarboxamido)-1,3-thiazole derivatives as antagonists for the adenosine A1 receptor. Bioorg. Med. Chem. Lett. 2001, 11, 2017-2019.

Vicini, P.; Geronikaki, A.; Incerti, M.; Busonera, B.; Poni, G.; Cabras, A. C.; Colla, L. P. Synthesis and biological evaluation of benzo $[d]$ isothiazole, benzothiazole and thiazole Schiff bases. Bioorg. Med. Chem. 2003, 11, 4785-4789.

Walek, W. Dichlorothiazolo[4,5-d]pyrimidines. Z. Chem. 1983, 23, 179-183 (Chem. Abstr. 1983, 99, 212485f).

Walek, W.; Pallas, M.; Goetzschel, K.; Kochmann, W.; Werner, R. D. Thiazolo[4,5-d]pyrimidines. Ger. (East) 1984, DD 208355 (Chem. Abstr. 1984, 101, 191965e).

Wobig, D. Thiazole derivatives: synthesis of thiazolo[4,5- $d]$ pyrimidine derivatives. Liebigs Ann. Chem. 1989, 409-411 (Chem. Abstr. 1989, 110, 212767v).

Xi, N.; Bo, Y.; Doherty, E. M.; Fotsch, C.; Gawa, N. R.; Han, N.; Hungate, R. W.; Klionsky, L.; Liu, Q.; Tamir, R.; et al. Synthesis and evaluation of thiazole carboxamides as vanilloid receptor 1 (TRPV1) antagonists. Bioorg. Med. Chem. Lett. 2005, 15, 5211-5217.

Zitouni, G. T.; Demirayak, A. O.; Kaplancikli, Z. A., Yildiz, T. M. Synthesis of some 2-[(benzazol-2-yl)thioacetylamino]thiazole derivatives and their antimicrobial activity and toxicity. Eur. $J$. Med. Chem. 2004, 39, 267-272.

Received May 14, 2011; accepted May 27, 2011 